\title{
A Method for Measuring Planar Residual Stresses in Rectangularly Orthotropic Materials
}

\author{
Charles W. Bert \\ School of Aerospace and Mechanical Engineering \\ University of Oklahoma, Norman, Okla.
}

AND

Gary L. ThOMPSON

Flutter and Structural Dynamics Group Aero Commander Division

North American Rockwell Corporation, Norman, Okla.

\begin{abstract}
A semidestructive method has been developed for determining the principal residual stresses and directions in rectangularly orthotropic materials. The reduction equations are based upon a set of functions that describe the surface strain-relaxation field about a hole drilled to a limited depth into the material. Three constants contained in the strain functions have to be determined by calibration tests; they are related to three general constants and the elastic material constants to establish applicability to an orthotropic material. Expressions for the planar residual-stress components in the material-symmetry directions are then developed, and from Mohr's stress circle, the principal residual stresses and directions are determined.
\end{abstract}

\section{INTRODUCTION}

$\mathbf{R}_{t}$ ESJDUAL STRESSES are quite often a nemesis for the engineer of today. When neglected in design, residual stresses can cause a part to fail below the design load or, under fatigue loadings, prior to the useful design life of the part. Residual stresses are sometimes neglected because they are not known to exist; at other times, they 
are neglected because their magnitudes and directions are unknown to the engineer and he has no knowledge of how to determine them. There is little hope for the first case. In the latter case, the cautious engineer usually incorporates a much larger safety factor than required resulting in a part that is over-designed, i.e. over-strengthened and over-weight.

Consequently, a number of different methods of measuring residual stresses have been developed. One of the earliest methods was the "boring out" method proposed by Sachs [1] in 1927 for circular-cross-section bars and tubes of macroscopically homogeneous isotropic materials. Recently this method was extended to cylindrically orthotropic bodies by Olson and Bert [2].

Another mechanical method was developed by Treuting and Read [3] in 1951. This method is applicable to plate-type parts and is based on a layer-removal technique that requires very precise grinding and measuring procedures to obtain accurate results. The method is restricted to very uniform stress fields. However, it can be used for orthotropic material providing all the assumptions and conditions can be met. One chief disadvantage of this method is that it is completely destructive. A specimen (say $4^{1 / 2}$ in. $x \frac{1}{2}$ in.) has to be removed from the part, thus rendering the part useless for its designed purpose.

In 1934 Mathar [4] originated a third mechanical method applicable to determination of planar residual stresses parallel with and adjacent to a flat surface on a part. This method, called the holedrilling method, is based on measurement of surface strain relaxation due to the drilling of a limited-depth hole normal to the surface. Care must be taken in the drilling of the hole as well as the location, installation, and reading of necessary strain gages. The method has been limited to macroscopically homogeneous, isotropic materials and calibration tests have had to be accomplished on specimens made of the same material for each investigation. These tests are limited in that the specimens cannot be loaded above a stress value of approximately one third the yield stress to allow for the stress concentration at the hole. In spite of the limitations, the hole-drilling method has been used widely since it is semidestructive. Quite often the hole is so small that it is acceptable. When it is not acceptable, it can sometimes be filled quite satisfactorily.

Recently the hole-drilling method was improved by Rendler and Vigness [5] by eliminating the necessity of a calibration for each material. This improvement was accomplished by assuming a certain functional form for the surface strain distribution. Two constants in the strain distribution must be determined by subsidiary experiments 
involving applied uniaxial stresses. These experiments were conducted for various ratios of hole depth to diameter and it was concluded that a ratio of 1.0 is the optimum, i.e. gives the largest strain response per unit stress. It was shown that the two constants determined for this ratio were valid for all isotropic elastic materials, and then measured strains can be related to the principal residual stresses. However, the analysis is restricted to macroscopically homogeneous, isotropic elastic materials, and as will be shown, the analysis neglected a factor which resulted in a small error.

Today many engineers work with more complicated materials, particularly fiber-reinforced composite materials, which behave anisotropically on a macroscopic basis. In this paper, the same approach as used in Ref. 5 is used in deriving the analytical relations required to determine the residual stresses in a rectangularly orthotropic material from hole-drilling strain measurements. The required calibration tests necessary for calculating the constants in the straindistribution functions are relatively simple tests and can be conducted on simple specimens in advance of determining the residual stresses.

In a residually stressed material, isotropic or not, the principal stress or principal strain directions are seldom known. Furthermore, Greszczuk [6] has pointed out that, in general, these directions do not coincide with one another. However, in most practical applications, if the material being investigated is known to be orthotropic, the material-symmetry directions are known and the associated elastic constants are known or can be determined experimentally. These elastic constants appear in the following stress-strain equations relating the surface strains in the material-symmetry directions $(1,2)$ to the corresponding stress components:

$$
\begin{aligned}
\epsilon_{11} & =\left(\sigma_{11} / E_{11}\right)-\left(\nu_{21} / E_{22}\right) \sigma_{22}, \\
\epsilon_{22} & =-\left(\nu_{12} / E_{11}\right) \sigma_{11}+\left(\sigma_{22} / E_{22}\right), \\
\epsilon_{12} & =\sigma_{12} / G .
\end{aligned}
$$

\section{THEORY}

Consider a material in a generalized-plane-stress field represented by the stress components $\sigma_{11}, \sigma_{22}, \sigma_{12}$ and orient a cylindrical coordinate system as shown in Figure 1. If a small circular hole is drilled normal to the surface to a small depth into the material, a change in surface strain will occur in the vicinity of the hole as a result of the stress relaxation associated with the drilling of the hole. The change 


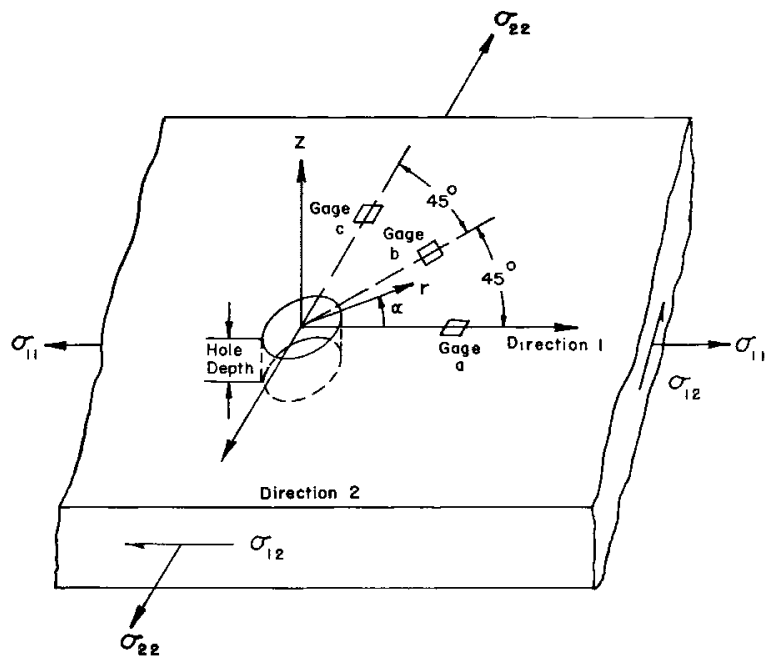

Figure 1. Schematic diagram showing coordinate system, stress components, hole geometry, and strain-gage locations.

in strain which occurs at any location at a fixed radial distance from the center of the hole can be expressed as

$$
\boldsymbol{\epsilon}(\alpha)=K(\alpha) \sigma_{11}+K\left(\alpha+90^{\circ}\right) \sigma_{22}+L(\alpha) \sigma_{12}
$$

where $\epsilon(\alpha)$ is the normal strain at some angle $\alpha$ measured from the major material-symmetry axis (direction 1), and $K(\alpha)$ and $L(\alpha)$ are strain distribution functions dependent on the angle $\alpha$.

If the strain functions $K(\alpha)$ and $L(\alpha)$ were known, it would be possible theoretically to establish a working expression by taking strain measurements at three unique orientations $\alpha$ and then solving for the three planar stress components. Of course, three separate single-element strain gages could be used, but it is more convenient to use one of the new three-element strain-gage rosettes with a central hole (especially designed for use in the hole-drilling method and now commercially available), as used by Rendler and Vigness [5].

The strain-distribution functions are assumed in the following forms:

$$
K(\alpha)=A+B \cos 2 \alpha, \quad L(\alpha)=C \sin 2 \alpha
$$

It is noted that this is the most general form of functions required to represent a homogeneous strain field. 
Substitutions of Eqs. (5) and (6) into Eq. (4) results in

$$
\begin{aligned}
\epsilon(\alpha) & =[A+B \cos 2 \alpha] \sigma_{11}+\left[A+B \cos \left(2 \alpha+180^{\circ}\right)\right] \sigma_{22} \\
& +[C \sin 2 \alpha] \sigma_{12}
\end{aligned}
$$

The suggested strain-gage array can be strategically oriented such that the strains $\epsilon_{a}, \epsilon_{b}$, and $\epsilon_{c}$ will correspond to $\alpha=0^{\circ}, 45^{\circ}$, and $90^{\circ}$ in the system being investigated.

If the values $\alpha=0^{\circ}, 45^{\circ}$, and $90^{\circ}$ are substituted into Eq. (7), the following three simultaneous equations result:

$$
\begin{gathered}
\epsilon_{a}=(A+B) \sigma_{11}+(A-B) \sigma_{22}, \\
\epsilon_{b}=A \sigma_{11}+A \sigma_{22}+C \sigma_{12}, \\
\epsilon_{c}=(A-B) \sigma_{11}+(A+B) \sigma_{22}
\end{gathered}
$$

These equations can be solved for $\sigma_{11}, \sigma_{22}$, and $\sigma_{12}$ in terms of $\epsilon_{a}, \epsilon_{b}$, and $\epsilon_{c}$ with the following results:

$$
\begin{gathered}
\sigma_{11}=\left[(A+B) \epsilon_{a}-(A-B) \epsilon_{c}\right] / 4 A B, \\
\sigma_{22}=\left[(A+B) \epsilon_{c}-(A-B) \epsilon_{a}\right] / 4 A B, \\
\sigma_{12}=\left(2 \epsilon_{b}-\epsilon_{a}-\epsilon_{c}\right) / 2 C
\end{gathered}
$$

In studying Eqs. (11)-(13) and comparing them with Eqs. (1)-(3), one might suspect that the constants $A, B$, and $C$ contain the elastic material constants. If general expressions could be developed for $A$, $B$, and $C$ in terms of the elastic constants, then Eqs. (11)-(13) would be applicable to an orthotropic, elastic material. Such expressions for $A, B$, and $C$ will be developed using an approach similar to the one used for isotropic material in Ref. 5 .

The strain components associated with the material-symmetry directions can be expressed in terms of the corresponding stresses and the elastic constants, provided the proper proportionality constants $\left(k_{1}, \ldots, k_{5}\right)$ are included, as follows:

$$
\begin{gathered}
\epsilon_{11}=\left(k_{1} \sigma_{11} / E_{11}\right)-\left(k_{2} \nu_{21} \sigma_{22} / E_{22}\right), \\
\epsilon_{22}=\left(k_{3} \sigma_{22} / E_{22}\right)-\left(k_{4} \nu_{12} \sigma_{11} / E_{11}\right), \\
\epsilon_{12}=k_{5} \sigma_{12} / G .
\end{gathered}
$$

In general, the five proportionality constants are required because the material being considered is orthotropic. However, definite relations do exist between the constants, and it will be shown that $A, B$, and $C$ depend on only three of the five constants. Also, it will be shown why a minimum of three proportionality constants are needed for isotropic materials, rather than only the two used in Ref. 5. 
Recognizing that $\epsilon_{a}$ and $\epsilon_{c}$ in Eqs. (8) and (10) are $\epsilon_{11}$ and $\epsilon_{22}$, one can compare these equations with Eqs. (14) and (15) and see that

$$
\begin{gathered}
A+B=k_{1} / E_{11}=k_{3} / E_{22}, \\
A-B=-k_{2} \nu_{21} / E_{22}=-k_{4} \nu_{12} / E_{11} .
\end{gathered}
$$

Also, from the Mohr's strain circle,

$$
\epsilon_{12}=2 \epsilon_{b}-\epsilon_{a}-\epsilon_{c}
$$

and from Eqs. (13) and (16), one contains

Thus,

$$
2 C=k_{5} / G \text {. }
$$

$$
\begin{gathered}
\left.A=(1 / 2)\left[k_{1} / E_{11}\right)-\left(k_{2} \nu_{21} / E_{22}\right)\right], \\
B=(1 / 2)\left[\left(k_{1} / E_{11}\right)+\left(k_{2} \nu_{21} / E_{22}\right)\right], \\
C=(1 / 2) k_{5} / G, \\
k_{3}=k_{1} E_{22} / E_{11}, \\
k_{4}=k_{2}\left(\nu_{21} / \nu_{12}\right)\left(E_{11} / E_{22}\right) .
\end{gathered}
$$

Therefore, only three independent constants $\left(k_{1}, k_{2}, k_{5}\right)$ exist. It should be noted that for isotropic materials, $E_{22}=E_{11}=E, \nu_{21}=\nu_{12}=\nu$, and $2(1+\nu) G=E$. For this case, $k_{3}=k_{1}$ and $k_{4}=k_{2}$ which agrees with Ref. 5. However, $C$ is not zero [since it equals $k_{5}(1+\nu) / E$ ], as was assumed in Ref. 5 . This explains why the predicted stresses obtained in Ref. 5 had a maximum error at $\alpha=45^{\circ}$ (where $\sin 2 \alpha=1.0$ and thus the effect of $C$ is greatest).

Therefore, if the elastic constants are known, one calibration to determine $k_{1}, k_{2}$, and $k_{5}$ in Eqs. (21)-(23) will suffice for a given orthotropic material.

\section{DETERMINATION OF EQUATION CONSTANTS BY CALIBRATION}

The original statement of the problem was based on the hypothesis that planar residual stresses in an orthotropic material could be determined by drilling a hole into the surface and measuring the surface-strain relaxation in the vicinity of the hole. In this paper, equations have been developed which relate the stresses in the material-symmetry directions of a rectangularly orthotropic material to the corresponding strain-relaxation values measured in the vicinity of the hole. In deriving these expressions, three constants occurred that may be determined readily by applying a known stress $\sigma$ in 
direction 1 to an orthotropic calibration specimen and allowing the stress in direction 2 to be zero. Denoting $\sigma_{11}$ as the applied stress $\sigma$ and recognizing that

$$
\epsilon_{a}=\epsilon_{11}, \epsilon_{c}=\epsilon_{22}
$$

Eqs. (8) and (10) can be solved for $A$ and $B$ with the following results:

$$
\begin{aligned}
& A=\left(\epsilon_{a}+\epsilon_{c}\right) / 2 \sigma \\
& B=\left(\epsilon_{a}-\epsilon_{c}\right) / 2 \sigma
\end{aligned}
$$

Thus, the constants $A$ and $B$ may be determined in terms of the known stress $\sigma$ and the radial strains $\epsilon_{a}$ and $\epsilon_{c}$ as measured at respective $\alpha$ angles of $0^{\circ}$ and $90^{\circ}$. To determine the constant $C$ appearing in Eq. (8) requires the application of a shear stress $\sigma_{12}$ in direction 1 or 2 , or a uniaxial normal stress at $\alpha=45^{\circ}$. For the case of a fairly homogeneous material, either isotropic or orthotropic, a uniaxial test at $45^{\circ}$ can be conducted successfully. However, this is not the case for fiber-reinforced composite materials, due to the problem of incomplete gripping of cut fibers. Thus, for such materials, either very specially designed uniaxial specimens [7] or in-plane shear loading must be used.

For the case of uniaxial loading at $45^{\circ}$, for instance,

$$
C=\epsilon_{b} / \sigma_{12}=2 \epsilon_{b} / \sigma
$$

During the calibrations, strain measurements at other angles should be taken. Then the assumed strain distribution can be checked by comparing the measured test values with the analytical ones determined from Eq. (4).

In Ref. 5, the optimal value of the ratio of hole depth to diameter was determined to be 1 . As the constants, $A, B$, and $C$ are functions of hole depth, a similar investigation would be in order when determining these constants for specific orthotropic materials. It would be expected that the optimal ratio of hole depth to diameter will be restricted to the particular orthotropic material used.

\section{GENERAL CALIBRATED EQUATIONS}

After selecting the optimal value for the ratio of hole depth to diameter, a proportional relation will exist between the associated constants $A^{\prime}$ and $B^{\prime}$, where the prime denotes the optimal values. This relation is defined to be

$$
B^{\prime}=\lambda A^{\prime}
$$


where $\lambda$ is a constant of proportionality.

Substituting this value of $B^{\prime}$ into Eq. (22) for $B$ and adding the resulting equation to Eq. (21) where $A$ has been appropriately replaced by $A^{\prime}$, one obtains

$$
A^{\prime}(1+\lambda)=k_{1} / E_{11} \text {. }
$$

Solving for $A^{\prime}$, one obtains:

$$
A^{\prime}=k_{1} /(1+\lambda) E_{11} \text {. }
$$

Substituting the value of $B^{\prime}$ from Eq. (31) into Eqs. (11) and (12) and again letting $A=A^{\prime}$, the results are:

$$
\begin{aligned}
& \sigma_{11}=\left[(1+\lambda) \epsilon_{a}-(1-\lambda) \epsilon_{c}\right] / 4 \lambda A^{\prime} \\
& \sigma_{22}=\left[(1+\lambda) \epsilon_{c}-(1-\lambda) \epsilon_{a}\right] / 4 \lambda A^{\prime}
\end{aligned}
$$

Substitution of $A^{\prime}$ from Eq. (32) yields

$$
\begin{aligned}
& \sigma_{11}=\left[(1+\lambda) \epsilon_{a}-(1-\lambda) \epsilon_{c}\right](1+\lambda)\left(E_{11} / 4 \lambda k_{1}\right) \\
& \sigma_{22}=\left[(1+\lambda) \epsilon_{c}-(1-\lambda) \epsilon_{a}\right](1+\lambda)\left(E_{11} / 4 \lambda k_{1}\right)
\end{aligned}
$$

and substitutions of Eq. (23) into Eq. (13) gives

$$
\sigma_{12}=\left(G / k_{5}\right)\left(2 \epsilon_{b}-\epsilon_{a}-\epsilon_{c}\right) \text {. }
$$

A numerical value of $k_{1}$ can be determined from Eq. (32) as the optimal values of $A^{\prime}$ and $B^{\prime}$ have been determined by calibration; a numerical value exists for $\lambda$, i.e. $B^{\prime} / A^{\prime}$. The major Young's modulus $E_{11}$ is known for the calibration material, and $k_{5}$ can be determined from Eq. (23) after calculating $C$ by the method described previously.

In these forms, Eqs. (35)-(37) represent the calibrated equations valid for determining the planar residual-stress system relative to material-symmetry directions of orthotropic materials.

\section{CALIBRATION CONSTANTS FOR ISOTROPIC MATERIALS}

The constants $A, B$, and $C$ have been shown to contain the material constants for isotropic materials as well as orthotropic materials. For isotropic materials, Rendler and Vigness [5] selected the optimal value of 1 for the ratio of hole depth to diameter and obtained corresponding values of $A^{\prime}$ and $B^{\prime}$ as follows:

$$
\begin{aligned}
& A^{\prime}=-0.225 \times 10^{-8} \mathrm{in.}^{2} / \mathrm{lb} . \\
& B^{\prime}=-0.450 \times 10^{-8} \mathrm{in.}^{2} / \mathrm{lb} .
\end{aligned}
$$

Since Ref. 5 showed a discrepancy in $\epsilon_{b}=\epsilon\left(45^{\circ}\right)$ of approximately 
-3 microstrain for the curve corresponding to $\epsilon_{a}=\epsilon\left(0^{\circ}\right)=-28$ microstrain and $\epsilon_{c}=\epsilon\left(90^{\circ}\right)=-9$ microstrain, the constant $C$ can be calculated as follows:

From Eqs. (28) and (30),

$$
C=2 \epsilon_{b} / \sigma=4 A^{\prime} \epsilon_{b} /\left(\epsilon_{a}+\epsilon_{c}\right)=-0.073 \times 10^{-8} \text { in. }^{2} / \mathrm{lb} .
$$

Introducing the elastic constants for cold-rolled steel, the dimensionless constants for all isotropic materials are:

$$
\begin{aligned}
& k_{1}=k_{3}=E\left(A^{\prime}+B^{\prime}\right)=\left(29.85 \times 10^{6}\right)\left(-0.675 \times 10^{-8}\right)=-0.201 \\
& k_{2}=k_{4}=-(E / \nu)\left(A^{\prime}-B^{\prime}\right)=-0.238 \\
& k_{5}=2 C G=2\left(-0.073 \times 10^{-8}\right)\left(11.64 \times 10^{6}\right)=-0.017
\end{aligned}
$$

Inserting the above values of the general constants into Eqs. (35)-(37) results in the following set of calibrated equations which are applicable to determination of planar residual-stress systems in any isotropic material with a hole drilled to a depth of one hole diameter:

$$
\begin{gathered}
\sigma_{11}=-1.86 E\left(3 \epsilon_{a}+\epsilon_{c}\right) \\
\sigma_{22}=-1.86 E\left(3 \epsilon_{c}+\epsilon_{a}\right) \\
\sigma_{12}=-58.8 G\left(2 \epsilon_{b}-\epsilon_{a}-\epsilon_{c}\right)
\end{gathered}
$$

\section{DETERMINATION OF THE PRINCIPAL RESIDUAL STRESSES}

If one can neglect the stresses acting in the $z$ direction (perpendicular to the drilled surface), and using the values for the stresses determined by Eqs. (35)-(37), one can use the Mohr's stress circle to determine the principal residual stresses as follows:

$$
\begin{aligned}
& \sigma_{\max }=\frac{\sigma_{11}+\sigma_{22}}{2}+\left[\left(\frac{\sigma_{11}-\sigma_{22}}{2}\right)^{2}+\sigma_{12}^{2}\right]^{1 / 2}, \\
& \sigma_{\min }=\frac{\sigma_{11}+\sigma_{22}}{2}-\left[\left(\frac{\sigma_{11}-\sigma_{22}}{2}\right)^{2}+\sigma_{12}^{2}\right]^{1 / 2}
\end{aligned}
$$

The stresses will be oriented at the following respective angles measured $C C W$ from the major material-symmetry axis (direction 1):

$$
\begin{aligned}
\tan \alpha_{\max } & =2 \sigma_{12} /\left(\sigma_{11}-\sigma_{22}\right), \\
\alpha_{\min } & =\alpha_{\max }+90^{\circ} .
\end{aligned}
$$




\section{NOMENCLATURE}

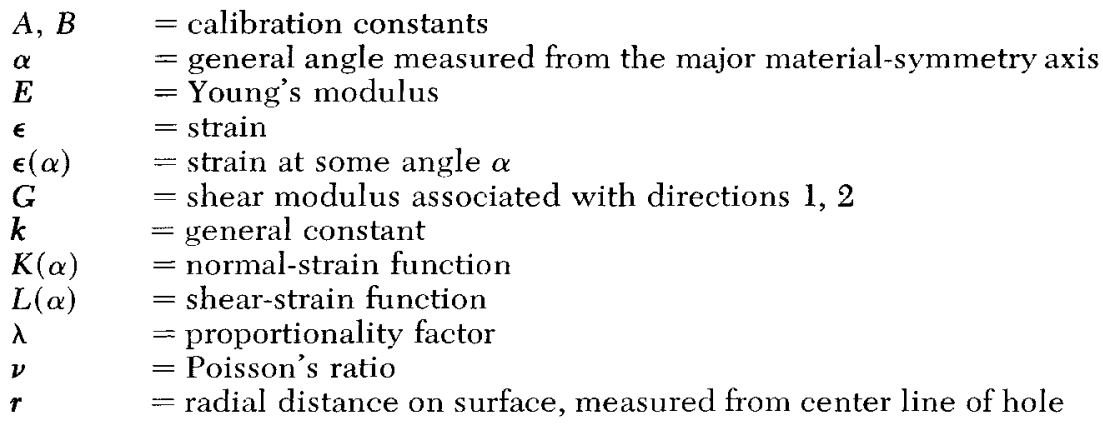

Subscripts

$a, b, c=$ rosette elements at $0,45,90$ degrees

$11,22=$ normal stress, normal strain, or elastic constants associated with directions 1,2

$12=$ shear stress or shear strain in direction 2 acting on face normal to direction 1

$1,2=$ major and minor material-symmetry directions

$1, \ldots, 5=$ designations for different general constants, $k$

$\max , \min =$ denotes principal stresses and associated directions

Superscripts

$=$ denotes optimal value

\section{REFERENCES}

1. G. Sachs, "Der Nachweiss Innerer Spannungen in Stangen und Rohren", Zeitschrift fur Metallkunde, Vol. 19 (1927), p. 352.

2. W. A. Olson and C. W. Bert, "Analysis of Residual Stresses in Bars and Tubes of Cylindrically Orthotropic Materials", Experimental Mechanics, Vol. 6 (Sept., 1966), p. 451 .

3. R. G. Treuting and W. T. Read, Jr., "A Mechanical Determination of Biaxial Residual Stress in Sheet Materials", Journal of Applied Physics, Vol. 22 (1951), p. 130.

4. J. Mathar, "Determination of Initial Stress by Measuring the Deformations Around Drilled Holes", Transactions of the A.S.M.E., Vol. 56 (1934), p. 249.

5. N. J. Rendler and I. Vigness, "Hole-Drilling Strain-Gage Method of Measuring Residual Stresses", Experimental Mechanics, Vol. 6 (December, 1966), p. 577.

6. L. B. Greszczuk, "Elastic Constants and Analysis Methods for Filament Wound Shell Structures", Missile \& Space Systems Division, Douglas Aircraft Company, Inc., Santa Monica, California, Report No. SM-45849 (January 1964).

7. V. G. Grinius, "Micromechanies-Failure Mechanism Studies", Air Force Materials Laboratory, Wright-Patterson AFB, Ohio, Report No. AFML-TR-66-177 (August 1966). 